\title{
LA TRADUCCIÓN Y LA HIBRIDACIÓN COMO PROBLEMAS PARA UNA INTERCULTURALIDAD AUTÓNOMA, COLABORATIVA Y DESCOLONIZADORA
}

\author{
Translation and Hybridization as Problems for Autonomous, Collaborative, and Decolonizing \\ Interculturality
}

\author{
Maya Lorena Pérez-Ruiz
}

Resumen: En la actualidad se vislumbran tres modelos de interacción entre los sistemas de conocimiento científico e indígena contenidos en la noción de interculturalidad: la perspectiva etnocéntrica y colonizadora, la perspectiva intercultural integradora, y la perspectiva intercultural autónoma, colaborativa y descolonizadora. No siemprese discuten las implicaciones que tienen la traducción de conocimientos y la hibridación en el diálogo intercultural. En este trabajo, a la luz de lo que se comprende como interculturalidad, se reflexiona sobre lo que ha sido la interacción asimétrica entre el sistema científico hegemónico y los sistemas indígenas subalternos, y se propone la construcción consensuada del sentido, los contenidos y las finalidades de la interculturalidad para avanzar hacia la superación de la perspectiva colonizadora, predominante en el diálogo de conocimientos.

Palabras clave: interculturalidad, cambio cultural, interacción cultural, construcción de conocimiento.

Abstract: Nowadays, three models of interaction show the possible relationship between scientific and indigenous knowledge systems: an ethnocentric colonial perspective, an intercultural integrating perspective, and an autonomous, collaborative, decolonizing intercultural perspective. Often those three models do not analyze how the translation of knowledge and hybridization influences intercultural dialogue. In this paper, through the lens of interculturality, we reflect on the asymmetric interaction between the hegemonic scientific and the subaltern indigenous systems; and we propose that the construction of meanings, contents and purposes of interculturality can be accomplished through consensual mechanisms in order to move beyond the colonizing perspective prevailing in dialogue about knowledge.

Keywords: interculturality, cultural change, cultural interaction, decolonization, construction of knowledge.

\footnotetext{
Maya Lorena Pérez Ruiz, doctora en Ciencias Antropológicas por la Universidad Autónoma de México, Unidad Iztapalapa. Investigadora de la Dirección de Etnología y Antropología Social, del Instituto Nacional de Antropología e Historia, México. Temas de especialización: pueblos indígenas, juventud, cultura, etnicidad, identidades, movimientos sociales y patrimonio cultural. Correo electrónico: mayaluum@gmail.com.
}

Enviado a dictamen: 22 de abril de 2015 Aprobación: 1 de octubre de 2015.

Revisiones: 1 . 


\section{Demandas indígenas e interculturalidad}

E n México, la lucha indígena por el reconocimiento de sus derechos ha tenido fases diferentes según la evolución de los pueblos indígenas como actores sociales, en el marco de sus interacciones con los poderes locales, nacionales y globales y de sus alianzas con otros actores sociales, lo que ha dado como resultado iniciativas y proyectos de diferentes índole y alcance. Primero se opusieron a la castellanización forzada, luego demandaron el derecho de ser educados en su lengua, además de que lucharon por el reconocimiento de sus conocimientos y culturas, y finalmente propusieron la autonomía como vía para construir formas alternativas de inserción dentro de la sociedad y el Estado nacionales. Esta perspectiva se alimentó de luchas latinoamericanas y anticoloniales (Pérez, 2009).

Tres momentos enriquecieron el movimiento indígena en México: en primer lugar, la Reunión de Barbados en 1971, en la que se criticó a los Estados nacionales y se defendió el derecho de los pueblos indígenas al autogobierno y a que ellos mismos protagonizaran sus luchas de liberación; en segundo lugar, el movimiento de resistencia indígena, negra y popular, previo a la conmemoración de los quinientos años del arribo de los europeos a América, que en México propició una intensa movilización indígena que contribuyó a que en 1990 se elevara a la Cámara de Diputados una iniciativa pionera para conseguir la autonomía regional pluriétnica y, posteriormente, a que en 1992 se modificara el artículo $4^{0}$ constitucional para reconocer los derechos culturales de los pueblos indígenas; y en tercer lugar el levantamiento del Ejército Zapatista de Liberación Nacional en 1994 que, por su alianza con el movimiento indígena nacional - aglutinado en la Asamblea Nacional Indígena Plural por la Autonomía-, hizo visible, nacional e internacionalmente, la lucha autonómica (Pérez, 2000 y 2005). ${ }^{1}$

Ante las luchas indígenas, el Gobierno mexicano respondió con estrategias múltiples que incluyeron la guerra de baja intensidad contra el EZLN y la sectorialización y oficialización de las demandas indígenas. Este proceso tuvo como resultados la desmovilización y la división del movimiento indígena - del que devinieron las reformas constitucionales de 2001 en materia de derechos indígenas, en las que se reconocía la autonomía indígena, pero sin sustento territorial y sin reconocer la pluralidad jurídica ni a los indígenas como sujetos de derecho (Pérez, 2005) así como la creación de la Coordinación General de Educación Intercultural Bilingüe, órgano de la Secretaría de Educación Pública promotor de la equidad y el desarrollo intercultural.

En elámbito latinoamericano, desde los años setenta, y con un repunte importante en la década de los noventa del siglo XX, también se produjeron movilizaciones indígenas que en algunos casos condujeron a la reformulación de los Estados nacionales para alcanzar sociedades más plurales, justas y equitativas. En estos procesos de lucha, la discusión de la interculturalidad se ha contemplado como opción para enfrentar la diversidad cultural. Ejemplos paradigmáticos son los Estados de Bolivia y Ecuador, que modificaron sus constituciones para reconocer los derechos ancestrales de los pueblos indígenas.

En este contexto, en la actualidad la noción de interculturalidad presenta una proliferación de usos y sentidos. Para algunos, la interculturalidad es un hecho social, un proceso que desde siempre se ha desarrollado cuando se encuentran e interactúan sujetos con culturas distintas, de ahí que algunos autores propongan desarrollar un campo de investigación intercultural que, de forma inter y transdisciplinaria, permita indagar sobre las tensiones entre la política, la cultura y la comunicación, presentes en las sociedades interculturales contemporáneas (García, 1995; Dietz, 2003; Dietz y Mateos, 2008). De este modo, la interculturalidad, al concebirse como parte de un proceso de interacción dialógica, puede concluir en la asimilación, o transculturación, como la denomina Zárate (2014). Este autor señala que una cultura se impone sobre otra engendrando colonialidad o, por el contrario, bajo los principios de respeto por la diferencia y la justicia, puede conducir a la autonomía, o hasta a la 
configuración de los pueblos indígenas como naciones, como sucede en Bolivia y Ecuador. Esto ocurre porque, si bien la noción de interculturalidad surge de medios académicos, ha sido apropiada y resignificada a la luz de las luchas indígenas por sus derechos.

En cambio, para otros autores el mero contacto o interacción entre individuos y grupos no desencadena procesos de interculturalidad, ya que la noción remite necesariamente a un tipo de relación e interacción en la que predominan la asimetría y la dominación (Aguirre Beltrán, 1967, 1976; De la Fuente, 1984). Por otra parte, otros autores, si bien reconocen que existen tales condiciones de subordinación, al referirse a la interculturalidad enfatizan el deber ser y la posibilidad de diálogo desde una posición ética y política, prescriptiva y normativa, que debe regir las interacciones entre individuos y grupos con culturas diferentes (Touraine, 1995), bajo principios de justicia, respeto y equidad. Se trata de lo que Walsh (2009) llama una interculturalidad crítica, que incumbe no sólo a los indígenas, sino a cualquier grupo social minorizado desde el poder a causa de sus características culturales, identitarias o raciales. Por estos motivos, la interculturalidad, como deber ser, con alcances diversos se discute para transformar la totalidad de los ámbitos de la vida social mediante: la educación bilingüe intercultural (Schmelkes, 2006 y 2009), la democracia intercultural (Krotz, 2004), los derechos humanos interculturales (Sierra, 2005), la autonomía intercultural (Díaz Polanco, 2005), el pluralismo jurídico (Walsh, 2002), la filosofía, la epistemología y la ontología intercultural (Villoro, 1998; Olivé, 1999; Fornet-Betancourt, 2003; Tubino, 2001) y la sociedad del conocimiento (Etskowitz y Leidesdorff, 1997). Asimismo, la interculturalidad también se disputa para proyectar nuevos Estados nacionales (De Sousa, 2007; Walsh, 2002 y 2009; Zambrano, 2005; Godenzzi, 2005). ${ }^{2}$ Estas propuestas cuestionan la interculturalidad oficializada, que mediatiza las demandas políticas y sociales y que, en el caso de los indígenas, disfraza su integración a proyectos nacionales y globales, asimétricos y colonizadores.
Ante este amplio campo de debate sobre la interculturalidad, en este trabajo importa desarrollar lo relativo a los conocimientos indígenas cuando sus creadores y portadores entran en interacción con los conocimientos científicos. Para fines analíticos, aquí se habla de forma simplificada de estos conocimientos para facilitar la comparación y el análisis del conflicto entre ellos. Por ello, no se pretende agotar la complejidad, la historicidad ni las desavenencias en el seno de las comunidades que delimitan, definen y validan estos tipos de conocimiento. ${ }^{3}$ Por conocimiento científico se entiende aquel que se define como tal desde los espacios de consagración académica, donde se construye y legitima este tipo de conocimiento, mediante criterios construidos en tales espacios, y según las características y parámetros de validación elaborados en un tiempo y un espacio específicos. De esta manera, pueden variar en el tiempo, y de un espacio académico a otro, según el momento histórico, la coyuntura social y los actores que acuerdan o disienten sobre los criterios para establecer lo que es y no es conocimiento científico. Para los fines de este artículo, los elementos que se ponderan dentro del conocimiento científico son la racionalidad, la objetividad y la universalidad, por ser los componentes que normalmente se emplean para descalificar los conocimientos indígenas, que se consideran locales, empíricos y cargados de significados míticos y religiosos, no objetivos. Se trata de principios positivistas que predominan en las relaciones de la ciencia hegemónica con otros sistemas de conocimiento, aun en espacios donde se construye la interculturalidad.

Al hablar de conocimientos indígenas se hace referencia a aquellos, también histórica, cultural y contextualmente situados, que han sido minorizados desde la ciencia hegemónica. Estos conocimientos han sido denominados de diferentes formas: sabiduría popular, saber local o folclore (Argueta, 1997 y 2011), ciencia indígena (De Gortari, 1963), ciencia de lo concreto (Lévi-Strauss, 1972), ciencias nativas (Cardona, 1979), conocimiento popular o ciencia del pueblo (Fals Borda, 1981 y 1988), conocimiento campesino (Toledo, 1994), etnoconocimientos y sistemas de saberes indígenas (Argueta, 1997 y 2011); 
local and indigenous knowledge systems (UNESCO, 2005) y traditional ecological knowledge (Johnson, 1992). Estos conceptos están siendo reposicionados al considerarse ya no como conocimientos inferiores, sino como epistemologías locales o epistemologías alternativas (Descola y Pálsson, 2001). Por sistemas de conocimiento indígenas se comprenden aquellos que los actuales pueblos indígenas consideran como propios, de acuerdo con la teoría del control cultural de Bonfil (1987), que reconoce procesos de imposición y expropiación en aquello que un pueblo considera propio, pero también de innovación y apropiación. Esto significa que tales sistemas de conocimiento no son estáticos ni idénticos a los de la época prehispánica, de forma tal que su evolución debe comprenderse bajo la tensión de la situación (Balandier, 1972), pero también dentro de los parámetros que los pueblos indígenas consideran válidos para seguirlos considerando como propios, a pesar de sus transformaciones y de que han adoptado elementos de otras culturas y sistemas de conocimiento.

Distingo cuatro aspectos que deben reflexionarse: a) el tipo de interculturalidad que enmarca la interacción entre sistemas de conocimiento; b) la caracterización inferiorizada de los conocimientos indígenas; c) la traducción como elemento fundamental para la interacción entre sistemas de conocimientos distintos, y d) la hibridación como medio y finalidad del diálogo intercultural.

\section{Los rostros de la interculturalidad}

Dada la proliferación de sentidos con que se aborda la interculturalidad, es fundamental dilucidar las principales vertientes de significado para aclarar sus similitudes y diferencias, incluso con el multiculturalismo, con el fin de comprender el tipo de interculturalidad que se requiere para establecer diálogos de conocimientos que no sean asimétricos ni subordinados.

El multiculturalismo nace en Estados Unidos en el siglo XX como respuesta al fracaso del modelo integrador melting-pot, que se cuestiona por soslayar la discriminación de las minorías mediante la homogeneización cultural reproducida mediante políticas de tradición liberal anglosajona, y que se sustenta, filosóficamente, en la concepción ilustrada de la tolerancia y en el principio de diferencia del liberalismo político. La tolerancia alude a una virtud ética de lo público: a la capacidad y la disposición de respetar las diferencias para no colocar las creencias propias como condición absoluta de la convivencia con el otro. Se propone distinguir la tolerancia negativa de la positiva. La primera se refiere a tolerar sin compartir en función de la convivencia, mientras que la tolerancia positiva propone situarse en el lugar del otro para comprender, desde dentro, las creencias y valoraciones del diferente. La tolerancia, como virtud cívica, es condición para la democracia liberal y es inseparable del ejercicio de la libertad de pensamiento y de la solidaridad social (Tubino, 2001). De este modo, el multiculturalismo anglosajón, como política pública, se propuso hacer operativa la tolerancia al incorporar a los diferentes asegurando su equidad en el ejercicio de los derechos reconocidos jurídicamente. Sin embargo, su incapacidad para hacer operativas las dos dimensiones de la tolerancia derivó en la opción de la interculturalidad, que enfatiza la tolerancia positiva, lo cual, en ese marco, es coherente con el pensamiento ilustrado y con el liberalismo político. Una crítica a la tolerancia multiculturalista la realiza Olivé (1999) al equipararla con un relativismo moral, problema en el que no incurre el pluralismo que él propone ya que, más que segregación de mundos y culturas, defiende la posibilidad de la pluralidad de mundos mediados por la comunicación para construir acuerdos racionales y para resolver así la inconmensurabilidad lingüística y cultural de grupos diferentes. De igual forma, Zizek (2008) cuestiona la tolerancia al pensarla como una forma astuta de desplazar la atención de la economía hacia la cultura y de permitir conductas abusivas, ante lo cual propone la intolerancia.

En América Latina, las agencias multilaterales y las organizaciones de cooperación fueron promotoras de las políticas educativas multiculturalistas, las cuales, según su perspectiva, fracasaron al propiciar la formación de islas étnicas bajo políticas tutelares. 
A cambio, se impulsó la interculturalidad en nuestros países (Tubino, 2001). Por su parte, Dietz (2003) deriva la interculturalidad del multiculturalismo anglosajón como medida para extender su influencia hacia América Latina que, en los años noventa, vivía un momento de posindigenismo. En esta región, según afirma, el multiculturalismo fue apropiado y reformulado bajo diversas influencias teóricas y empíricas, de ahí que los discursos interculturales derivados de tal perspectiva sean transnacionales, con acentos continentales, nacionales y regionales, producidos por la hibridación y la fertilización de las diferentes tradiciones disciplinarias de los protagonistas (Dietz y Mateos, 2008).

Tienen otra perspectiva quienes consideran que en América Latina se usó la noción de interculturalidad con un sentido diferente a la de multiculturalismo porque existió una convergencia temporal en el uso de esta noción, aunque no en sus objetivos. Abona a esta perspectiva recordar que Aguirre Beltrán usó la noción de interculturalidad desde mediados del siglo XX para explicar la organización territorial y social, que pone en relación de simbiosis económica, y parasitaria, a la población mestiza de una ciudad rectora de una amplia región geográfica, con las comunidades indígenas que están dentro de ese hinterland; un tipo de dominación arcaica que se expresa en la explotación económica de los mestizos sobre los indígenas. Para este autor, en la condición intercultural se enfrentan culturas diferentes, pero también se influyen al ser la aculturación un conflicto de fuerzas en el que se desarrollan tanto procesos de adopción, como de reinterpretación, e inclusive de contra-aculturación (Aguirre Beltrán, 1967 y 1970). Por otra parte, en el ámbito de la educación Emilio Monsonyi fue quien formuló con claridad la propuesta para transitar de la educación bicultural, predominante dentro del indigenismo de integración, hacia la educación intercultural. ${ }^{4}$

Autores como Juan C. Godenzzi (2005), Luis Enrique López (2001 y 2007) y Fidel Tubino (2005, 2014), entre otros, se preocupan también por establecer las diferencias entre una y otra forma de interculturalidad, según provenga del multiculturalismo o de la perspectiva latinoamericana. Para Godenzzi (2005), una preocupación relacionada con el multiculturalismo consiste en hacer compatible el liberalismo - basado en el principio de igualdad - con el derecho a la diferencia; de ahí la importancia de temas como el universalismo, el particularismo, la igualdad, la diferencia, los derechos colectivos, la justicia etnocultural, las políticas de reconocimiento y la supuesta neutralidad del Estado moderno frente a la diversidad cultural. Esta perspectiva también para Walsh (2009) y Fornet-Betancourt (2003) es parcial y políticamente interesada puesto que, al limitarse a aspectos político-simbólicos o meramente culturales, omite analizar las bases materiales y sociohistóricas de la asimetría y de la subordinación en que entran en conflicto los individuos y grupos con culturas diferentes. Otro aspecto relevante para marcar las diferencias es que la educación multiculturalista y luego intercultural, en países anglosajones y luego europeos, atañe únicamente a la población inmigrante y no a las poblaciones originarias, cuya lucha por los derechos tiene peculiaridades que derivan del hecho de ser pueblos colonizados (Bonfil, 1972, 1991). De este modo, al proponer facilitar el cruce de fronteras y la convivencia entre grupos diferentes, se trabaja en beneficio de la sociedad envolvente para la asimilación ya que, en condiciones de dominación, la síntesis o hibridación cultural es producto de la erosión, y hasta de la destrucción, de la cultura subordinada (López, 2001; Walsh, 2009; Tubino, 2014).

En cambio, la perspectiva latinoamericana de lo intercultural, si bien se ha aplicado mayoritariamente al campo de la educación, en su actualización política y a la luz de los nuevos movimientos sociales y de la influencia de los enfoques postcoloniales ya no se limita a establecer medidas que faciliten la asimilación de la diversidad cultural, comprenda ésta a los pueblos indígenas, a las comunidades afroamericanas o a cualquier otra cultura subalterna, como lo hace el multiculturalismo en diversos grados y formas según el país. De este modo pretende no sólo lograr el reconocimiento de las diferencias culturales o crear métodos educativos que propicien mejores formas de interacción e integración, sino apuntar a transformar las condiciones y modalidades en las que se dan las 
relaciones e interacciones sociales para acabar con la dominación y propiciar la liberación cultural, así como de la política y la economía. Estos esfuerzos, en perspectivas radicales de transformación se encaminan a refundar los Estados nacionales en un marco de justicia social, cultural y epistémica y sobre la base del reconocimiento del derecho a la diferencia y a la pluralidad, como proyecto en el que es posible que existan y se relacionen mundos diferentes bajo acuerdos políticos (Olivé, 1999; FornetBetancourt, 2003; Godenzzi, 2005; Walsh, 2009).

Respecto de los problemas educativos, López (2001) percibe similitudes entre la propuesta de educación intercultural acuñada en América Latina y la empleada por algunos países europeos - que han generado nuevas propuestas por la creciente inmigración de población que llega de otros continentes-, ya que en algún punto ambas contemplan el enriquecimiento mutuo a través del intercambio cultural. Sin embargo, las diferencias estribarían en los propósitos de la interacción, del diálogo y del aprendizaje cultural ya que, para la perspectiva europea continental, postmulticulturalista, el objetivo sería construir espacios de encuentro para la hibridación cultural, para que mediante el intercambio cultural y el aprendizaje mutuo se diluyeran las fronteras hasta construir nuevas identidades. Por otro lado, en el contexto latinoamericano la educación intercultural tendría entre sus propósitos saldar deudas históricas con las poblaciones indígenas originarias y, por tanto, trataría de conseguir un diálogo respetuoso desde la diferencia para, desde el fortalecimiento identitario de los subordinados, negociar y regular los intercambios y el enriquecimiento de cada una de las culturas en términos de equidad.

Entre ciertos movimientos indígenas, como los de Bolivia y Ecuador, la transformación que se pretende para el Estado nacional tiene un propósito anticolonial que rebasa también los límites de la interculturalidad educativa asociada con el multiculturalismo anglosajón y europeo. Les critican omitir las condiciones estructurales de la desigualdad, la inequidad, la asimetría o la exclusión, lo que propicia una visión culturalista del conflicto de la diferencia y encubre la subordinación estructural. Además, estos movimientos consideran la interculturalidad educativa como desmovilizadora de la acción colectiva al promover - bajo la bandera de la interculturalidadla integración y la hibridación, lo que conduce soterradamente a la renuncia de las identidades y de las culturas de los grupos subordinados, operando a favor del proyecto modernizador neoliberal y globalizador. Esta perspectiva, reformulada desde parámetros decoloniales, es apoyada desde ámbitos académicos (Walsh, 2009), pero también cuenta con observaciones que alertan sobre los riesgos de asumirla acríticamente sin atender a las mediaciones que incluyen aspectos históricos, de poder y de hegemonías, y sin asumir la crítica de las nociones de cultura, interculturalidad y decolonización (Estermann, 2014).

Reconocer qué tipo de interculturalidad se propone y cómo en ella se enuncia y se desarrolla la interacción entre sistemas de conocimiento distintos es fundamental para dilucidar el propósito último de la interacción intercultural. Se trata de una reflexión indispensable ya que en nuestros países, bajo la influencia de la interculturalidad multiculturalista y en concordancia con los planes gubernamentales dirigidos a controlar las movilizaciones sociales, se observa un avance sustantivo de esta vertiente mediante políticas e instituciones públicas; incluso la interculturalidad latinoamericana ha sido domesticada y folclorizada y se ha vuelto funcional al sistema global de corte neoliberal y a los Estados nacionales, asimétricos en lo social y económico y homogeneizantes e integradores en lo cultural (Tubino, 2005; Walsh, 2009). Esta perspectiva avanza peligrosamente en los espacios de educación intercultural, oficiales y no oficiales de México, en los que, incluso bajo un discurso radical sobre los fines y los medios de la interculturalidad, persiste la hegemonía de los conocimientos científicos. De este modo, los conocimientos indígenas sirven para "enriquecer", "complementar" y dar carácter local y "étnico" a los conocimientos científicos, en tanto que la "cultura indígena" sirve para marcar pertenencias identitarias, lo mismo que para amenizar festivales y ceremonias oficiales. 
La supremacía del conocimiento científico sobre los conocimientos indígenas

En nuestros países, a lo largo de más de quinientos años la mayoría de las interacciones entre los conocimientos no indígenas y los indígenas han estado marcadas por el conflicto y por la supremacía de los primeros sobre los segundos. De la misma forma, desde la hegemonía de la ciencia contemporánea los conocimientos indígenas han sido descalificados, perseguidos y destruidos, pero también han sido expropiados selectivamente para enriquecer el conocimiento científico o para su comercialización, ajena a las comunidades que los crearon. Así, a través de la historia los pueblos indígenas han padecido la destrucción, la sustitución y la expropiación de sus conocimientos.

A pesar de ello, paulatinamente se avanza hacia el reconocimiento de los conocimientos indígenas, lo que en el campo académico se refleja en la proliferación y consolidación de las etnociencias (Argueta, 1997 y 2010), en las cuales, sin embargo, prevalece lo "etno" como marca de otredad y minorización. En estas disciplinas, además, prevalece la traducción y la secularización de los conocimientos indígenas para liberarlos de los elementos incómodos, generalmente religiosos y míticos incomprensibles para la razón científica, de modo que puedan ser entendidos, comparados y validados por el conocimiento científico mediante sus referentes de veracidad. Afortunadamente, también se avanza desde la reflexión epistemológica, ética y jurídica en una tendencia que privilegia el valor de la diversidad y la pluralidad y que cuestiona los enfoques monoculturales creados desde los diferentes ámbitos de poder.

En cuanto a la discusión filosófica, son ejemplos significativos Villoro (1998), Olivé (1999) y FornetBetancourt (2003), quienes desde la pluralidad proponen la construcción de nuevas formas de hacer filosofía, de pensar la epistemología y de situarse frente al pensamiento indígena y sus conocimientos. Así, Fornet-Betancourt (2007) asume los retos que la perspectiva intercultural ha implicado para la filosofía latinoamericana, al confrontarla por su apego a la filosofía hegemónica europea y al obligarla a repensarse en la situación colonial y postcolonial. El reto es que ésta se conciba como una filosofía histórica y contextualmente situada, que permita asumir como materia filosófica la diversidad cultural, la pluralidad y los desafíos que esto conlleva. Lo anterior conduce a comprometerse con las poblaciones indígenas en sus luchas por lograr su derecho a la cultura y su autodeterminación económica, política y religiosa. Para este autor, no puede haber interculturalidad sin liberación de las alteridades. Y, en ese camino, la filosofía intercultural debe contribuir a pluralizar el ethos de los derechos humanos y de la idea de democracia, así como a fomentar una pedagogía del reconocimiento del otro y a universalizar la crítica contra los fundamentalismos, incluido el de Occidente y su lógica económica y de conocimiento.

Villoro (1998) apoya también el fin de la idea moderna de la unidad universal de la razón, gestada bajo la racionalidad moderna de Occidente. En cambio, propone comprender la razón en su diversidad como resultado de una multiplicidad inagotable de culturas. Asume también la opción de construir Estados plurales que incluyan la diversidad de identidades culturales, la autonomía y un universalismo ético desde el cual comprender el relativismo cultural y abrir caminos hacia nuevas formas de vida y organización social. En una línea similar, Olivé (2004) propone la construcción de una sociedad multicultural con apoyo pluralista en la filosofía, en la ética y en la epistemología para poner fin a las posiciones absolutistas, y aun relativistas, extremas.

Otro campo desde el que se debate la pluralidad epistemológica asociada con los conocimientos indígenas es el de la sociedad del conocimiento: una etapa nueva de las sociedades contemporáneas cuyo proyecto societal se desarrolla económica y socialmente sobre el soporte del conocimiento científico - teórico y tecnológico- y de las tecnologías de la información y la comunicación. De este modo, el recurso principal de explotación es el capital intelectual bajo un modelo de producción del conocimiento en el que se diluyen las fronteras disciplinarias. Tiene como característica una aceleración sin precedentes en la producción, distribución y capitalización del conocimiento, pero 
también en su depreciación. En este sentido, bajo la propuesta de la "triple hélice" (Etzkowitz y Leidesdorff, 1997) se pone a trabajar a las universidades, a las empresas y a los gobiernos para la innovación y la competitividad que exigen los mercados internacionales para el desarrollo, supuestamente al servicio del bienestar económico y social de todos. En la práctica, es un modelo excluyente e impositivo que conlleva prácticas asimétricas e inequitativas, tanto entre naciones como entre comunidades científicas, $y$, por supuesto, hacia otras formas de conocimiento, como los indígenas. Y, si bien algunos autores proponen una vía intercultural para la sociedad del conocimiento (como Olivé, por ejemplo), para los críticos de lo que se trata es de incorporar los "otros" conocimientos y tecnologías al ámbito de la ciencia y la tecnología científica bajo el eufemismo de que será para el bien de todos. De ahí la importancia de la divulgación científica, la validación y la incorporación de los saberes tradicionales a las lógicas de competitividad y de mercado.

Un ejemplo de tal perversión se observa en la UNESCO que, si bien promueve la sociedad del conocimiento de forma pluralista - que incluye los derechos humanos y que debe regirse sobre los principios de libertad de expresión, acceso a la educación, acceso a la universalidad de la información y respeto a la diversidad cultural y lingüística-, omite los contextos de fuerte asimetría y desigualdad presentes en el mundo contemporáneo. Además, los saberes tradicionales serán importantes en la medida en que sean compatibles con los tipos de desarrollo y de mercados impulsados por la lógica neoliberal de la globalización. En los hechos, los conocimientos que denomina tradicionales son inferiorizados desde el momento en que deben ser traducidos, subsumidos, hibridizados eintegrados a los sistemas de conocimiento científicos para el "bien de la humanidad" (UNESCO, 2014b), y sin un beneficio explícito para los creadores de tales conocimientos. Se trata de una fórmula de incorporación de los conocimientos indígenas a las políticas globales del conocimiento científico y del desarrollo neoliberal que no discute el carácter expropiatorio de tal incorporación ni cuestiona las condiciones que perpetúan la subalternidad de quienes produjeron esos conocimientos.

\section{La traducción como subalternidad}

En el siglo XX, cuando la antropología, la biología, la agronomía y otras disciplinas evidenciaron las potencialidades de los sistemas de conocimiento indígenas para organizar y clasificar suelos y plantas, para manejar y conservar ecosistemas complejos, o para identificar material botánico y animal útil para la medicina y la alimentación, se reforzó la necesidad de comparar, de "traducir" y de encontrar equivalencias de manera unilateral entre los conocimientos indígenas y los desarrollados por la ciencia, lo que acentuó la expropiación de saberes, que incluso fueron patentados por especialistas y empresas (Pérez y Argueta, 2011).

Hasta hoy, y aun en los espacios de construcción de la interculturalidad, se privilegia la necesidad de traducir y validar los conocimientos indígenas, para la integración y la hibridación, dentro de los esquemas hegemónicos estipulados por la ciencia. De ahí que persista la visión subordinada que enmarca el encuentro entre los saberes indígenas y los institucionalizados en centros educativos y de investigación. Más allá de las buenas intenciones dirigidas a generar diálogos interculturales simétricos, en la interacción entre sujetos de ámbitos culturales distintos persisten operaciones de reconocimiento desiguales que ponen en marcha los procesos históricos y contextuales mediante los cuales se ha interiorizado la inferiorización de lo indígena.

Se trata de procesos cognitivos mediante los cuales los sujetos descifran, categorizan, adscriben, evalúan, clasifican y califican los atributos de los otros sistemas de conocimiento que resultan externamente evaluados. Esta evaluación se impone por el dominante desde el ejercicio de su poder académico, económico, político, cultural, lingüístico y simbólico (Giménez, 2003) y, al presentarse como un hecho objetivo, se oculta la construcción social de la diferencia, es decir, los procesos de valoración y clasificación en virtud de los cuales se asignan - e imponen - las cualidades y atributos. 
Según Poutignat y Streiff-Fenart (1995), el hecho de la nominación, de nombrar y calificar al otro, en contextos de asimetría y dominación, constituye un aspecto revelador de las relaciones interétnicas en la medida en que se evidencia uno de los mecanismos de la etnización, al tiempo que en sí mismo es un hecho productor de etnicidad por su poder preformativo. Esto significa que el hecho de nombrar tiene el poder, inclusive, de hacer existir a una colectividad de individuos mediante diversos mecanismos sociales sin que importe que tales individuos o grupos se autoconciban de otra forma, ni que se opongan a la valoración que se les impone, como la de ser indios, grupos étnicos, hispanos, afrodescendientes o asiáticos, entre muchas otras denominaciones subalternas (Pérez, 2007).

En ese sentido, la minorización y la estigmatización de los conocimientos indígenas hecha desde la ciencia como saber hegemónico está detrás de que sean calificados como empíricos, orales y de alcance limitado, al suponer que carecen de métodos de validación objetivos, así como de formas de experimentación, sistematización y transmisión, puesto que se cree que se adquieren de forma casi natural a través de los sentidos y mediante prácticas cotidianas de prueba y error. De esta manera, se niegan los componentes de cualquier proceso cognitivo y se desconocen las formas complejas que durante miles de años los diversos pueblos originarios han desarrollado para generar, comprobar, sistematizar, transmitir y conservar sus sistemas de conocimiento. ${ }^{5}$ Estas cosmovisiones y sistemas de conocimiento, en ciertos momentos históricos y bajo ciertas condiciones de poder y hegemonía, igual que sucede ahora con los conocimientos científicos, también se constituyeron como universales frente a sus propios subalternos en amplias áreas culturales.

Un paso para dejar atrás la subalternidad del conocimiento indígena es el que propone Olivé (2004 y 2007), para quien el diálogo de saberes presupone la operación de fuerzas racionales en una interacción comunicativa, lo que implica una disposición para escuchar y para actualizarse mediante un intercambio de conocimientos, apreciaciones y valores en los que operan fuerzas racionales para la interacción comunicativa. Se basa en reconocer que existe la razón como capacidad humana distinta al concepto de racionalidad, vista desde el ejercicio de la razón humana en un contexto cultural e histórico. Dentro del pluralismo epistemológico, la objetividad, entendida como aceptabilidad racional, se supone presente en todos los sistemas cognitivos, ya sea que se agrupen bajo un mismo marco conceptual o que se construyan sobre condiciones epistémicas específicas y dentro de diálogos interculturales particulares. Con dicho diálogo no se pretendería el relativismo extremo ya que el pluralismo epistemológico propone construir contextos propicios para entablar acuerdos interpretativos dirigidos a encontrar los elementos que conduzcan hacia metas comunes para la resolución de problemas concretos. Esta propuesta enfatiza la interpretabilidad conjunta y acordada, más que la traducción, como vía para superar la inconmensurabilidad entre marcos conceptuales y visiones del mundo diferentes. Esto supone avanzar hacia lo propuesto por De Sousa Santos (2007), en términos de emprender procesos epistemológicos -interculturales e interepistémicos- que deben suceder sobre la base de una "justicia cognitiva" y de una ontología múltiple.

Otro paso más será abandonar la caracterización del conocimiento indígena inferiorizada e impuesta desde el poder, la cual ha sido asumida acríticamente por los indígenas como efecto de la subordinación para, en los términos de Olivé vistos antes, recaracterizar y revalorar los conocimientos indígenas, así como crear contextos que propicien una interacción no asimétrica ni colonial entre actores con sistemas de conocimiento distintos.

Lo anterior, sin embargo, no resuelve de forma automática el problema de caracterizar el proceso, los resultados y la finalidad que tendrán las interacciones del diálogo intercultural. ¿Es suficiente y pertinente emplear la noción de hibridación? ¿No se trata de una noción que oculta los procesos de selección de lo que se desecha o se adopta, además de que omite desentrañar el qué, el cómo y el para qué del diálogo intercultural? 


\section{Sobre el rostro oculto de la hibridación}

En la actualidad, la hibridación se emplea como una fórmula fácil para dar cuenta del resultado de la interacción entre sujetos con culturas distintas, de forma que sustituye a términos como mestizaje, sincretismo, aculturación y transculturación, entre otros que han sido empleados para explicar los procesos de mezcla, cambio e incorporación de elementos culturales a lo que se tenía antes. Pero, iqué se entiende por hibridación? ¿Y en beneficio de quién y para qué se lleva a cabo?

En términos generales, la noción de hibridación ha sido ampliamente empleada en los estudios culturales y literarios para señalar el origen mixto y disímil de lo que ocupa espacios intersticiales, con mezclas, propiciando el mestizaje y la emergencia de nuevas identidades sincréticas o entremezcladas. La propusieron diversos autores vinculados con el postmodernismo para, junto con las nociones de juego, fluidez, fusión y rizoma, dejar atrás visiones estáticas o esencialistas sobre las identidades y las culturas y con ello desdibujar las fronteras y los límites estáticos entre lo culto, lo masivo y lo popular, que estuvieron vigentes para caracterizar los procesos culturales durante varias décadas del siglo XX (Barfield, 2000). En América Latina, el gran impulsor del concepto de hibridación fue Néstor García Canclini a partir de su libro Culturas híbridas (1990). Desde entonces, bajo la evidencia de que todas las culturas son híbridas - mezclas - , su uso se expandió profusamente en las ciencias sociales, en ocasiones para mencionar, más que para explicar, complejos procesos culturales.

No obstante, la noción de hibridación se ha criticado severamente porque, con su aparente neutralidad y aleatoriedad, oculta y disfraza procesos sobre los que deben tomarse posiciones éticas y políticas. En América Latina la han cuestionado Gilberto Giménez (2002), Carlos Reynoso (2000) y Pablo Alabarces (2012), entre otros. Cuestionan su poca capacidad heurística ya que es fundamentalmente un concepto descriptivo, no esclarece procesos ni construye explicaciones sobre el qué, el cómo, el por qué y bajo qué relaciones de poder se dan los procesos específicos de interacción, intercambio e imposición cultural en que se produce la hibridación. Al tratarse de una metáfora no explicativa, sin connotación de orden, composición o dominancia no analiza, en contextos específicos, ni la interiorización ni la subjetividad que induce a los actores a incorporar unos elementos culturales y a desechar otros, de forma que al emplear la noción de hibridación se usurpa el papel de la teoría y se sustituye por una engañosa y superficial evidencia empírica (Giménez, 2002).

Por parte de los movimientos indígenas en América Latina también existe desconfianza en torno a la noción de hibridación ya que, como proceso y finalidad de la interculturalidad, se asocia con las políticas multiculturalistas, concordantes con proyectos filosóficos, económicos y políticos del neoliberalismo que, bajo la metáfora de la mezcla, pretenden la pérdida de las identidades indígenas, ocultan la asimilación, y se oponen a las luchas autonómicas que buscan la reconstitución de los pueblos que han sido colonizados, con sus identidades y culturas propias. Si con todo lo anterior se decide por la hibridación, los parámetros de qué, cómo, para qué y en beneficio de quiénes ha de darse deberían establecerse mediante procesos de acuerdo político, ético y epistemológico para que se decidieran los procesos y límites de la hibridación en términos de equidad y de justicia en prevención de sus consecuencias.

\section{Reflexiones finales}

Como se ha dicho previamente, en la actualidad existen tres posibilidades de interacción entre los sistemas de conocimiento científico y los indígenas: la perspectiva etnocéntrica y colonizadora, la perspectiva intercultural integradora y la perspectiva intercultural autónoma, colaborativa y descolonizadora (Pérez y Argueta, 2011), y en cada una de ellas tienen un sentido distintivo la traducción y la hibridación.

La primera perspectiva es etnocéntrica y colonizadora porque propone la interacción entre ciencia y conocimientos indígenas para enriquecer los sistemas de conocimiento hegemónicos y, en esa medida, se propone 
la destrucción abierta o encubierta de los sistemas indígenas ya que para fines propios genera procesos de expropiación, a la par que realiza la secularización, la traducción, la validación y el uso de aquellos conocimientos que considera útiles para la ciencia o para bien de los intereses comerciales y turísticos en proyectos afines con el desarrollo del capitalismo global.

La segunda, la intercultural integradora, si bien habla de diálogo intercultural, lo hace con la finalidad de romper las fronteras identitarias y, bajo el eufemismo de "hibridizarse mutuamente", actúa en beneficio de los actores, identidades y sistemas de conocimiento dominantes al no desactivar los mecanismos de poder y hegemonía, al perpetuar las relaciones de subordinación hacia la otredad y al reproducir los procesos subjetivos de interiorización de la subalternidad. Para tal diálogo se requiere también de la secularización, la traducción y la validación de conocimientos en beneficio del sistema expropiador y receptor $y$, aunque se pretende la horizontalidad, la balanza se inclina a favor de los actores que sustentan el poder y la capacidad institucional para crear, sistematizar, aplicar y reproducir los nuevos conocimientos "híbridos" creados.

En cuanto a la tercera perspectiva, la intercultural autónoma, colaborativa y descolonizadora, ésta postula la autonomía de los sistemas de conocimientos como condición para el diálogo, para que los actores implicados participen desde sus lógicas culturales y cosmogónicas en condiciones de respeto y equidad, dentro de los espacios establecidos y acordados para la interacción. En términos de Olivé (1999, 2004 y 2007) y de De Sousa Santos (2007), se pretendería poner en marcha el pluralismo epistemológico, la justicia cognitiva y la ontología múltiple para construir contextos propicios dirigidos a entablar acuerdos interpretativos a fin de construir metas comunes. En este caso, la apropiación selectiva de elementos culturales, lo mismo que la exportación de elementos considerados propios hacia el otro sistema de conocimiento, tendrían que ser acordados, porque la finalidad del proceso intercultural no sería la destrucción de ambos sistemas para construir algo nuevo, diferente a los puntos de partida, sino fortalecer con autonomía ambos sistemas de conocimiento.
Importa aclarar que no se propone que los sistemas de conocimiento deban permanecer aislados, puros, para ser autónomos. En cambio, se afirma que la autonomía es una condición política que incluye derechos y capacidades para garantizar la autoreproducción y el desarrollo de los grupos cultural y socialmente subordinados; una condición dirigida a la interacción horizontal entre sistemas de conocimiento distintos, en donde los pueblos indígenas, ahora subordinados, tengan la capacidad de decidir sobre sus procesos de permanencia y de cambio, así como sobre las apropiaciones e innovaciones que consideran que deben tener sus pueblos, y sus sistemas de conocimiento, mediante el diálogo intercultural. Este requisito contribuiría a evitar la imposición y la expropiación desde el poder, sea éste científico, político, económico o simbólico.

Un diálogo de conocimientos bajo la tercera opción no supone, por tanto, que el conocimiento científico pierda su propia lógica para asumir la del conocimiento indígena, ni tampoco que los sistemas indígenas pierdan sus elementos ontológicos distintivos para que se secularicen y asuman la lógica y la perspectiva de la ciencia. No implica discutir la validez ontológica, o la eficacia y la capacidad explicativa de un sistema de conocimiento en comparación con otro, sino que cada actor ha de acudir al diálogo desde su epistemología propia y dentro de los espacios y finalidades acordados para la interacción entre sistemas de conocimiento, evitando la traducción unilateral e impositiva, para construir de manera consensuada los significados de aquello que se dispondrá para el diálogo intercultural.

Para que un diálogo así sea posible deben resolverse problemas como: las dificultades de la ciencia para reconocer y aceptar el estatus epistemológico de otros sistemas de conocimiento, como los indígenas; la omisión del plano estructural que encubre las asimetrías, el conflicto y la subordinación entre sistemas de conocimiento, y la peligrosa convergencia de la lucha autonómica indígena con las políticas neoliberales de reconocimiento de sesgo multiculturalista, que omiten las condiciones estructurales de desigualdad social y cultural en el conjunto de las relaciones sociales. 
En ese proceso, además, se tendrán que superar aspectos como: las perspectivas incorporacionista e integracionista, que promueven la renuncia del desarrollo autónomo de los sistemas de conocimiento indígenas en beneficio de una hibridación impuesta; el utilitarismo y la secularización en la selección del tipo de conocimientos deseables para el diálogo intercultural; el discurso de la modernización y del desarrollo mundial, globalizado e integrador con visión multiculturalista, que subyace como proyecto para el diálogo intercultural; así como la segregación y la resistencia como vías ante la falta de transformaciones sustantivas para el diálogo intercultural colaborativo y anticolonial. Todo ello requiere de voluntad política y de acuerdos entre diversos actores.

Optar por una de las tres perspectivas señaladas antes implica una decisión política y ética conjunta para desvelar los rostros ocultos de la interculturalidad integradora.

\section{Notas}

${ }^{1}$ En Pérez (2005) analicé las organizaciones indígenas en alianza o en confrontación con el EZLN, que difieren según sus proyectos y su posición frente a la autonomía y el Estado nacional: las reivindicativas, políticas o antisistémicas.

${ }^{2}$ En Pérez (2009) se exploran las aportaciones de cada uno de estos autores.

${ }^{3}$ Ver Olivé (1999) sobre el constructivismo realista y plural que, basado en Kuhn, se opone al mismo tiempo al relativismo extremo y devastador.

${ }^{4}$ Lo hace en 1983, en la reunión continental sobre el Mayor Proyecto de Educación en América Latina y el Caribe, convocada por la UNESCO y el Instituto Indigenista Interamericano (Monsonyi y Rengifo, 1983; López, 2001; Tubino, 2005).

5 Díaz Tepepa et al. (2011) demuestra la falacia del empirismo en los conocimientos campesinos.

\section{Referencias bibliográficas}

Aguirre Beltrán, Gonzalo (1967). Regiones de refugio El desarrollo de la comunidad y el proceso dominical en Mestizo-América. México: Instituto Indigenista Interamericano.

Aguirre Beltrán, Gonzalo (1976). Obra polémica. México: SEP-INAH

Alabarces, Pablo (2012). "Transculturas pospopulares. El retorno de las culturas populares en las ciencias sociales latinoamericanas". En Cultura y Representaciones sociales, año 7, núm. 13, septiembre, pp. 7-39. Disponible en: www.culturayrs.org.max/revista (consultado en marzo de 2013).

Argueta Villamar, Arturo (1997). Epistemología e historia de las etnociencias. Tesis de Maestría en Ciencias, Facultad de Ciencias, UNAM, México.

Argueta Villamar, Arturo (2010). "Los sistemas de saberes indígenas y el diálogo intercultural”. En M. Gómez (ed.), Reflexiones sobre la sociedad del conocimiento y la interculturalidad en México. México: UNAM, pp. 9-23.

Argueta Villamar, Arturo (2011). "El diálogo de saberes, una utopía realista". En Arturo Argueta Villamar, Eduardo M. Corona y Paul Hersch (coord.), Saberes locales y diálogo de saberes. México: CRIM, UNAM, pp. 495-510.

Balandier, Georges (1972). El concepto de 'situación colonial'. México: ENAH.

Barfield, Thomas (ed.) (2000). Diccionario de antropología. México: Siglo XXI.

Bonfil Batalla, Guillermo (1972). "El concepto de indio. Una categoría de la situación colonial”. En Anales de Antropología, vol. IX, pp. 106-124. México: UNAM.

Bonfil Batalla, Guillermo (1987). La teoría del control cultural en el estudio de los procesos étnicos. México: CIESAS.

Bonfil Batalla, Guillermo (1991). "Los conceptos de diferencia y subordinación en el estudio de las culturas populares". En Pensar nuestra cultura. México: Alianza Editorial, pp. 58-67. 
Cardona, G. Raimondo (1979). “Categorías cognoscitivas y categorías lingüísticas en huave”. En I. Italo Signorini et al., Los huaves de San Mateo del Mar. México: INI, pp. 315-348.

De Gortari, Eli (1963). La ciencia de la historia en México. México: Fondo de de Cultura Económica.

De la Fuente, J. (1984). Educación, antropología y desarrollo de la comunidad. México: INI-SEP.

De Sousa Santos, Boaventura (2007). La reinvención del Estado y el Estado plurinacional. Cochabamba: CENDA, CEJIS, CEDIB.

Descola, Philippe y Gísli Pálsson (coord.) (2001). Naturaleza y sociedad, perspectivas antropológicas. México: Siglo XXI.

Díaz Polanco, Héctor (2005). "Los dilemas de la diversidad". En Revista de Interculturalidad, año 1, núm.l, octubre 2004-marzo 2005, pp. 45-62.

Díaz Tepepa et al. (2011). "Innovar en la tradición. La construcción local de los saberes campesinos en procesos interculturales". En Arturo Argueta, Eduardo Corona y Paul Hersch (eds.), Saberes colectivosy diálogo de saberes en México. México: UNAM-CRIM, pp. 235-124

Dietz, Gunther (2003). Multiculturalismo, interculturalidad y educación: una aproximación antropológica. Granada, España: Universidad de Granada.

Dietz, Gunther y Laura Mateos (2008). El discurso intercultural ante el paradigma de la diversidad. México: UAM-Iztapalapa.

Esterman, Josef (2014). “Colonialidad, descoloniación e interculturalidad". En Polis. Revista Latinoamericana, núm. 30. Disponible en: http://polis.revues.org/10164 (consultado en mayo de 2015).

Etzkowitz, Henry y Loet Leidesdorff (ed.) (1997). Universities and the Global Knowledge Economy: a Triple Helix University-Industry-Government. Londres y Washington: Printer.

Fals Borda, Orlando (1981). "La ciencia del pueblo". En Investigación participativa y praxis rural. Nuevos conceptos en educación y desarrollo comunal. Lima: Editorial Mosca Azul, pp. 19-47.

Fals Borda, Orlando (1988). Ciencia propia y colonialismo intelectual. Los nuevos rumbos. Bogotá: Carlos Valencia Editores.
Fornet-Betancourt, Raúl (2003). "Interculturalidad: asignatura pendiente de la filosofía latinoamericana. Para una revisión crítica de la filosofía latinoamericana reciente". México: Asociación de Filosofía y Liberación. Disponible en: http://www.afyl.org/ txt/Articulos/RaulFornet_Interculturalidad.pdf (consultado en marzo de 2009).

Fornet-Betancourt, Raúl (2007). "La filosofía intercultural desde la perspectiva latinoamericana”. En ElSolar, núm. 3, año 3, Lima, pp. 23-40. Disponible en: htpp://www. revistasolar.org.pe/3/03_betancourt.pdf (consultado en marzo de 2009).

García Canclini, Néstor (1990). Culturas hibridas. Estrategias para entrar y salir de la modernidad. México: Grijalbo, CNCA

García Canclini, Néstor (1995). Consumidores y ciudadanos. Conflictos multiculturales de la globalización. México: Grijalbo.

Giménez, Gilberto (2002). “iCulturas híbridas en la frontera norte?" En Fátima Flores (coord.), Senderos del pensamiento social. México: UNAM, Ediciones Coyoacán, pp. 37-52.

Giménez, Gilberto (2003). "Las diferentes formas de discriminación desde la perspectiva de la lucha por el reconocimiento social". Disponible en: www. cultuayrs.org.mx/seminario/bibliografía (consultado en abril de 2011).

Godenzzi, Juan Carlos (2005). “Introducción. Diversidad histórica y diálogo intercultural. Perspectiva latinoamericana”. En Tinkuy. Boletín de Investigación y Debate, núm. 1, invierno, Sección de Estudios de la Universidad de Montreal. Disponible en: www.littlm.umontreal.ca/documents/REVISTA TINKUY_l_o00doc (consultado en marzo de 2009).

Johnson, M. (1992). Lore. Capturing Traditional Enviromental Knowledge. Ottawa: Dene Cultural Institute, International Development Research Centre.

Krotz, Esteban (2004). "Los derechos humanos hoy: de la aculturación al diálogo intercultural". En Milka CastroLucic (ed.), Losdesafios de la interculturalidad. Identidad, política y derecho. Santiago: Universidad de Chile, pp. 151-172.

Levi-Strauss, C. ([1962]1972). El pensamiento salvaje. México: Fondo de Cultura Económica. 
López, Luis Enrique (2001). "La cuestión de la interculturalidad y la educación latinoamericana". En Séptima Reunión del Comité Regional Intergubernamental del Proyecto Principal de Educación en América Latina y el Caribe, UNESCO. Disponible en: www.aulaintercultural.org/IMG/pdf/ Lopez-UNESCO.pdf (consultado en marzo de 2009).

López, Luis Enrique (2007). "Diversidad cultural, multilingüismo y reinvención de la educación intercultural bilingüe en América Latina”. En Teresa Durán, Desiderio Catruquir y Arturo Hernández (comp.), Patrimonio cultural Mapuche, vol. II. Temuco, Chile: Universidad Católica de Temuco.

Monsonyi, Esteban Emilio y Francisco Rengigo (1983) "Fundamentos teóricos y programáticos de la educación intercultural bilingüe". En Nemesio Rodríguez et al. (ed.), Educación, etnias y descolonización en América Latina, vol. 1. México: UNESCO, pp. 209-230.

Olivé, León (1999). Multiculturalismo y pluralismo. México: Paidós.

Olivé, León (2004). Interculturalismoy justicia social. México: Programa Nación Multicultural-UNAM.

Olivé, León (2007). La ciencia y la tecnología en la sociedad del conocimiento. México: Fondo de Cultura Económica.

Pérez Ruiz, Maya Lorena (2000). "Pueblos indígenas, movimientos sociales y lucha por la democracia". En Estado del desarrollo económico y social de los pueblos indígenas de México, t. I. México: INI, pp. 355-418.

Pérez Ruiz, Maya Lorena (2005). iTodos somos zapatistas! Alianzas y rupturas entre el EZLN y las organizaciones indígenas de México (1994-2001). México: INAH.

Pérez Ruiz, Maya Lorena (2007). "El problemático carácter de lo étnico". En CUHSO: Cultura/Hombre y Sociedad, núm. 13. Temuco, Chile: Centro de Estudios Socioculturales de la Universidad Católica, pp. 35-55.

Pérez Ruiz, Maya Lorena (2009). “iDe qué hablamos cuando nos referimos a lo intercultural?" En Laura Valladares, Maya Lorena Pérez Ruiz y Margarita Zárate Vidal (coord.), Estados plurales. Los retos de la desigualdad y la diferencia. México: UAM-Iztapalapa y Juan Pablos Editor, pp. 251-288.

Pérez Ruiz, Maya Lorena y Arturo Argueta Villamar (2011). "Saberes indígenas y diálogo intercultural". En
Cultura y Representaciones Sociales, año 5, núm. 10, pp. 3156. México, IIS-UNAM,

Poutignat Philippe y Jocelyne Streiff-Fenart (1995) Théories de l'ethnicité. París : Presses Universitaires de France.

Reynoso, Carlos (2000). Apogeo y decadencia de los estudios culturales. Una visión antropológica. Barcelona: Gedisa.

Schmelkes, Sylvia (2006). "La interculturalidad en la educación Básica". Ponencia presentada en la Segunda Reunión del Comité Intergubernamental del Proyecto Regional de Educación para América Latina y el Caribe, UNESCO, Santiago de Chile, del 11 al 13 de julio de 2006.

Schmelkes, Sylvia (2009). "Interculturalidad. Democracia y formación valoral en México". En Revista Electrónica de Investigación Educativa, vol. 11, núm. 2. Disponible en: http://redie.uabc.mx/index.php/redie/article/view/233 (consultado en marzo de 2010).

Sierra, María Teresa (2005). "Diálogos y prácticas interculturales: derechos humanos, derechos de la mujeres y políticas de identidad". En Revista de Interculturalidad, año 1, núm. 1, octubre 2004-marzo 2005, pp. 63-88

Toledo, Víctor. M. (1994). La apropiación campesina de la naturaleza: un análisis etnoecológico. Tesis de doctorado en Ciencias, Facultad de Ciencias, UNAM, México.

Touraine, Alain (1995). "¿Qué es una sociedad multicultural? Falsos y verdaderos problemas". En Claves de Razón Práctica, núm. 56, pp. 14-25.

Tubino Arias-Schreiber, Fidel (2001). "Interculturalizando el multiculturalismo". En Intercultural. Balance y perspectivas. Encuentro Internacional sobre Interculturalidad. Barcelona: Fundación CIDOB. Disponible en: www. cidob.org (consultado en marzo de 2009).

Tubino Arias-Schreiber, Fidel (2005). "La praxis de la interculturalidad en los Estados nacionales latinoamericanos". En Cuadernos Interculturales, juliodiciembre, vol. 3, núm. 5, Universidad de Valparaíso, Viña del Mar, pp. 83-96. Disponible en: http:// redalyc.uaemex.mx/redalyc/pdf/552/55200506.pdf (consultado en marzo de 2009).

Tubino Arias-Schreiber, Fidel (2014). "El trasfondo epistémico de los conflictos interculturales". En 
Revista Contextualizaciones, año 6, núm. 1l, juliodiciembre, pp. 1-5. México: Departamento de Estudios Ibéricos y Latinoamericanos-Universidad de Guadalajara. Disponible en: http://www. contextualizacioneslatinoamericanas.com.mx/pdf/El transfondoepist $\%$ C3\%A9micodelosconflictosinterc ulturales_ll.pdf (consultado en marzo de 2015).

UNESCO (2005). Local and Indigenous Knowledge Systems. S.l.: UNESCO. Disponible en: http://portal.unesco. org (consultado en marzo de 2015).

UNESCO (2014a). "Sistemas de conocimientos locales e indígenas". S.l: UNESCO. Disponible en http:// www.unesco.org/new/es/natural-sciences/priorityareas/links/related-information/what-is-local-andindigenous-knowledge/ (consultado en marzo de 2015).

UNESCO (2014b). Conocimientos locales y tradicionales y políticas de CTI". Montevideo: Oficina de la UNESCO en Montevideo. Disponible en: www.unesco.org/new/ es/office-in-montevideo/ciencias-naturales/cienciatecnologia-e-innovacion/conocimientos-locales-ytradicionales-y-politicas-de-cti/ (consultado en marzo de 2015).
Villoro, Luis (1998). Estado plural, pluralidad de culturas. México: Paidós, UNAM.

Walsh, Catherine (2002). "Interculturalidad, reformas constitucionales y pluralismo jurídico”. En Boletín ICCI-RIMAI, Publicación Mensual del Instituto Científico de Culturas Indígenas, año 4, núm. 36, marzo, Bogotá. Disponible en: http://icci.nativeweb.org/boletin/36/ walsh.html $\not 1$ (consultado en mayo 2015).

Walsh, Catherine (2009). "Interculturalidad crítica y educación intercultural". Ponencia presentada en el Seminario Interculturalidad y Educación intercultural, organizado por Instituto Internacional de Integración Andrés Bello, marzo, La Paz, Bolivia.

Zárate Pérez, Adolfo (2014). "Interculturalidad y decolonialidad". En Tabula Rasa, núm. 20, enero-junio, pp. 91-107.

Zambrano, Carlos V. (2005). "Interculturalidad, reconocimiento y diversidad ampliada". En Revista Interculturalidad, año 1, núm. 1, octubre 2004-marzo 2005. Santiago, Chile, pp. 89-106.

Zizek, Slavoj (2008). En defensa de la intolerancia. Madrid: Ediciones Sequitur. 